\title{
Oxytocin receptors in the dorsolateral bed nucleus of the stria terminalis (BNST) bias fear learning toward temporally predictable cued fear
}

Daisy Martinon ${ }^{1}$, Paulina Lis ${ }^{1}$, Alexandra N. Romann ${ }^{1}$, Patricio Tornesi ${ }^{1}$, Sarah V. Applebey ${ }^{1}$, Garrett Buechner ${ }^{1}$, Valentina Olivera ${ }^{1,2,3}$ and Joanna Dabrowska (1),2,3

\begin{abstract}
The inability to discriminate between threat and safety is a hallmark of stress-induced psychiatric disorders, including post-traumatic stress disorder. Dorsolateral bed nucleus of the stria terminalis (BNST $T_{d \mid}$ ) is critically involved in the modulation of fear and anxiety, and has been proposed to regulate discrimination between signaled (cued, predictable) and unsignaled (unpredictable) threats. We recently showed that oxytocin receptors (OTRs) in the BNST dl facilitate acquisition of cued fear measured in a fear-potentiated startle (FPS). In the current study, using in vivo microdialysis in awake male Sprague-Dawley rats, a double immunofluorescence approach with confocal microscopy, as well as retrograde tracing of hypothalamic BNST-projecting OT neurons, we investigated whether fear conditioning activates OT system and modulates OT release. To determine the role of OTR in fear memory formation, we also infused OTR antagonist or OT into the BNST $d$ before fear conditioning and measured rats' ability to discriminate between cued (signaled) and non-cued (unsignaled) fear using FPS. In contrast to acute stress (exposure to forced swim stress or foot shocks alone), cued fear conditioning increases OT content in BNST dl microdialysates. In addition, fear conditioning induces moderate activation of OT neurons in the paraventricular nucleus of the hypothalamus and robust activation in the supraoptic and accessory nuclei of the hypothalamus. Application of OT into the BNST dl facilitates fear learning toward signaled, predictable threats, whereas blocking OTR attenuates this effect. We conclude that OTR neurotransmission in the BNST dl plays a pivotal role in strengthening fear learning of temporally predictable, signaled threats.
\end{abstract}

\section{Introduction}

Oxytocin (OT) is a hypothalamic peptide, hormone, and a neuromodulator, first isolated and then synthesized by Vincent du Vigneaud ${ }^{1}$, who later received Nobel Price for his work. OT receptor (OTR) in a G-protein-coupled

\footnotetext{
Correspondence: Joanna Dabrowska (joanna.dabrowska@rosalindfranklin.edu) 'Department of Cellular and Molecular Pharmacology, Chicago Medical School, Rosalind Franklin University of Medicine and Science, North Chicago, IL 60064, USA

${ }^{2}$ Center for the Neurobiology of Stress Resilience and Psychiatric Disorders, Rosalind Franklin University of Medicine and Science, North Chicago, IL 60064, USA

Full list of author information is available at the end of the article.
}

receptor, which can propagate signal transduction via either $\mathrm{G \alpha}_{\mathrm{i}}$ or $\mathrm{G \alpha} \alpha_{\mathrm{q}}$ proteins, activate a variety of signaling cascades ${ }^{2}$. In addition to regulating reproductive function and water/electrolyte homeostasis, OT modulates a wide range of fear and anxiety-like behaviors; for review, see refs. ${ }^{2,3}$. Although substantial evidence suggests that OT has anxiolytic properties ${ }^{4-6}$, the role of OT neurotransmission in the regulation of conditioned fear appears more complex and is brain region specific ${ }^{7-9}$. Some conflicting data on the role of OT in the regulation of fear responses might stem from the fact that the great majority of behavioral studies utilize exogenous OT application to

\section{(c) The Author(s) 2019}

(c) (i) Open Access This article is licensed under a Creative Commons Attribution 4.0 International License, which permits use, sharing, adaptation, distribution and reproduction cc. in any medium or format, as long as you give appropriate credit to the original author(s) and the source, provide a link to the Creative Commons license, and indicate if changes were made. The images or other third party material in this article are included in the article's Creative Commons license, unless indicated otherwise in a credit line to the material. If material is not included in the article's Creative Commons license and your intended use is not permitted by statutory regulation or exceeds the permitted use, you will need to obtain permission directly from the copyright holder. To view a copy of this license, visit http://creativecommons.org/licenses/by/4.0/. 
define its biological function, whereas the role of endogenous OT in anxiety and fear formation is largely unknown. In a fear-potentiated startle (FPS), systemic OT reduces background anxiety without affecting cued or contextual fear ${ }^{10,11}$. In the FPS, cued fear is measured as a potentiation of the startle amplitude to startle-eliciting noise during presentations of conditioned stimuli $\left(\mathrm{CS}^{+}\right)$, which have been previously paired with foot shocks. Background anxiety (non-cued fear) reflects potentiation of the startle measured between the $\mathrm{CS}^{+}$presentations. Importantly, non-cued fear recall depends on the initial $\mathrm{CS}^{+}$presentation, as it is not observed until after the $\mathrm{CS}^{+}$ presentations ${ }^{12}$, and is mainly independent of contextual fear ${ }^{10,11}$. Therefore, cued and non-cued fear responses can be used to determine rats' ability to discriminate between signaled (cued) and unsignaled (diffuse) stimuli, as described before ${ }^{13}$.

The dorsolateral bed nucleus of the stria terminalis $\left(\mathrm{BNST}_{\mathrm{dl}}\right)$ is a key brain area for translating stress into sustained anxiety ${ }^{14-16}$. Imaging studies in humans have shown potentiation of the BNST activity in conditions of uncertainty ${ }^{17}$, during hypervigilant threat monitoring ${ }^{18}$, and in anticipatory anxiety in participants suffering from arachnophobia ${ }^{19}$. The activity of the BNST is further exaggerated in patients suffering from anxiety disorders $^{17,18}$. In animal models, BNST lesions disrupt expression of contextual fear ${ }^{20}$, as well as conditioned fear responses to long-lasting cues ${ }^{16,21}$, but not to short, discrete cues ${ }^{22-24}$. However, growing evidence suggests that the BNST is also involved in the modulation of conditioned fear responses to discrete cues ${ }^{25,26}$. BNST lesion improves ability to discriminate between cues paired with unconditioned stimuli (US) vs. unpaired cues ${ }^{27}$. Recent studies confirm the involvement of the BNST in learning to discriminate between CS representing safety and CS representing threat ${ }^{28}$, phasic vs. sustained $\mathrm{fear}^{29}$, and signaled vs. unsignaled threats ${ }^{13,25}$.

The BNST has one of the highest expression levels of $\mathrm{OTR}^{30-33}$ and receives OT inputs, at least partly, from the paraventricular nucleus of the hypothalamus $(\mathrm{PVN})^{7,30}$. We recently demonstrated that OTR neurotransmission in the $\mathrm{BNST}_{\mathrm{dl}}$ facilitates the acquisition of conditioned fear to a discrete cue ${ }^{12}$. Here we show that OT is selectively released in the $\mathrm{BNST}_{\mathrm{dl}}$ during cued fear conditioning, highlighting the involvement of endogenous $\mathrm{OT}$ in cued fear learning. Moreover, we demonstrate that fear conditioning induces robust activation of OT neurons in the accessory (AN) and supraoptic nuclei of the hypothalamus $(\mathrm{SON})$, and that both of the nuclei project to the $\mathrm{BNST}_{\mathrm{dl}}$. Finally, using in vivo pharmacology and FPS, we calculate discrimination indices of individual rats by comparing a proportion of cued with non-cued fear as before $^{13}$. We show that OTR transmission in the BNST $_{\mathrm{dl}}$ facilitates discrimination learning between cued (signaled, predictable) and non-cued (unsignaled, unpredictable) fear, whereas blocking OTR attenuates this discrimination. Our results show that OTR in the $\mathrm{BNST}_{\mathrm{dl}}$ biases fear learning toward the formation of adaptive fear responses of cued, signaled, predictable threats.

\section{Methods and materials \\ Animals}

Male Sprague-Dawley rats (Envigo, Chicago, IL; 240-300 g) were housed in groups of three on a $12 \mathrm{~h}$ light/dark cycle with free access to water and food. Protocols for animal experiments in this study were performed in accordance with the guidelines of the National Institute of Health and approved by the Animal Care and Use Committee at Rosalind Franklin University of Medicine and Science.

\section{The effect of fear conditioning, acute stress, or social interactions on OT content in BNST $d$ microdialysates Fear conditioning}

A total of 31 rats were used in the experiment (Supplementary Table 1), 6 rats were eliminated due to missing microdialysate samples, and 2 rats were eliminated due to misplacement of the probe. Microdialysis in freely moving rats was performed as before ${ }^{34}$ (Supplementary Methods 1.1-1.2).

All experiments were conducted in SR-LAB startle chambers (San Diego Instruments, San Diego, CA) ${ }^{12}$ (Supplementary Methods 1.3.1). On any experimental day, baseline microdialysis samples were collected from three rats. After $5 \mathrm{~min}$ of acclimation in SR-LAB chambers, one experimental rat received ten presentations of a $3.7 \mathrm{~s}$ cue light (CS), each co-terminating with a $0.5 \mathrm{~s}$ foot shock (US; $0.5 \mathrm{~mA}$, inter-trial interval 60-180 s). Another rat placed in a neighboring chamber received the same ten foot shocks without the CS. Samples from the third control rat, placed in a Plexiglas microdialysis cage, were continuously collected in the same room (Fig. 1a). After three baseline microdialysis samples' collection, fearconditioning session began in parallel with the fourth sample collection. Session started with 5 min acclimation, continued for $20 \mathrm{~min}$, and the rats remained inside the chambers for additional $5 \mathrm{~min}$ after the session ended, after which the microdialysate sample was collected $(100 \mu \mathrm{l})$. The rats were then returned to their microdialysis cages and four more samples were collected in $30 \mathrm{~min}$ intervals $(100 \mu \mathrm{l}$ each).

\section{Forced swimming}

There were 41 rats used in the forced swimming (FS) and social interaction (SI) experiments, and 9 rats were excluded from the analysis (misplacement or inability to confirm probe placement). After baseline samples collection, rats were placed in Plexiglas tanks filled with 
A. OT release in the BNST via in vivo microdialysis

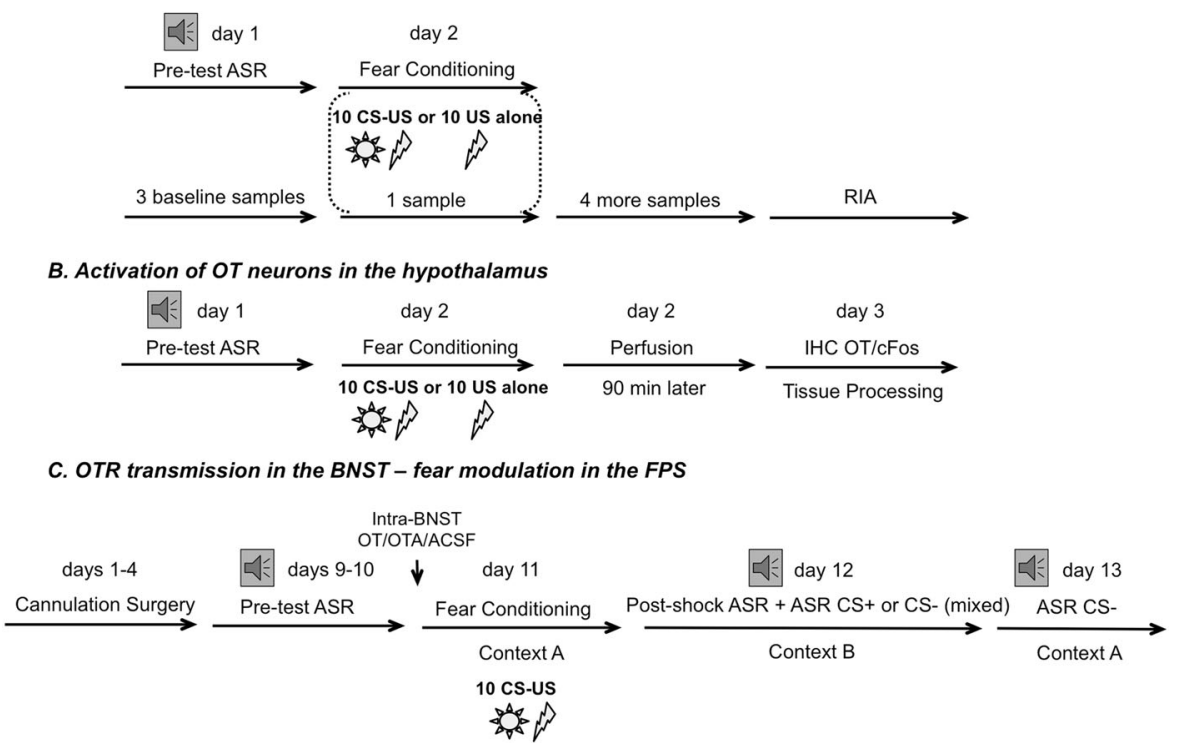

Fig. 1 Schematic representation of the experimental design (a-c). a Rats were habituated to the chambers and tested for an acoustic startle

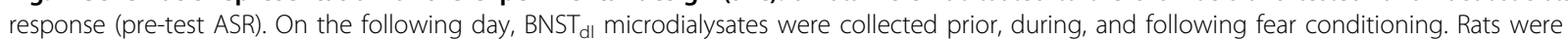
subjected to either cue lights paired with foot shocks (CS-US), or unsignaled foot shocks (US alone). Control rats were placed inside the microdialysis cages without a light or shock exposure. b Following exposure to foot shocks signaled by a cue, unsignaled foot shocks, or control conditions (exposure to cue alone or no cue, no foot shock exposure) rats were perfused and hypothalamic sections were processed for double immunofluorescence labeling with antibodies against OT and marker of immediate early gene expression, cFos. c Before cued fear conditioning (CSUS), cannulated rats were infused bilaterally into the BNST dl with OT, oxytocin receptor antagonist (OTA), or artificial cerebrospinal fluid (ACSF) in context A. Twenty-four hours later, rats were tested for the recall of cued and non-cued fear in context B. The recall test consisted of 10 habituation ASR trials (excluded from analysis), followed by ASR measured during the presence $\left(\mathrm{CS}^{+}\right)$or absence of cue light, mixed in a pseudorandom order (40 trials). Twenty-four hours later, rats were tested for the contextual fear recall (ASR measured without $\mathrm{CS}^{+}$presentations) in context $\mathrm{A}$

water for $10 \mathrm{~min}^{35}$. They were then returned to microdialysis cages and five more microdialysis samples were collected (Supplementary Methods 1.3.2).

\section{Social interactions}

After three baseline sample collections, a novel rat was placed in the microdialysis cage with the experimental rat for $10 \mathrm{~min}$ (Supplementary Methods 1.3.3).

\section{Probe placement and radioimmunoassay for OT}

Radioimmunoassay was performed as before ${ }^{34}$ (see inclusion criteria in Fig. 2 legend and Supplementary Methods 1.4-1.5).

\section{The effect of fear conditioning on activation of hypothalamic OT neurons \\ Fear conditioning and timely perfusions}

Ninety minutes following exposure to unsignaled foot shocks, foot shocks signaled by a cue, or control conditions ( $n=5$ per condition), rats were deeply anesthetized with Somnasol (Henry Schein Animal Care, Dublin, $\mathrm{OH}$ ) and transcardially perfused with $10 \%$ buffered formalin. In another cohort, rats were exposed to cue light alone or control conditions ( $n=3$ per condition) and were perfused as above. Brains were then sliced $(50 \mu \mathrm{m})$ on a SM2000R sliding microtome (Leica Biosystems, Nussloch, Germany) and processed for double immunofluorescence for OT and immediate early gene expression, $\mathrm{cFos}^{30}$ (Fig. 1b, Supplementary Methods 2.1).

\section{Confocal microscopy and cells counting}

An FV10i confocal laser-scanning microscope (Olympus, Waltham, MA) was used to acquire high-resolution $Z$-stack images ( $\times 60$ magnification at $1 \mu \mathrm{m}$ interval), which were taken bilaterally from the PVN, SON, and AN (both medial and lateral AN when applicable) of every third hypothalamic brain section from the entire brain $(150 \mu \mathrm{m}$ interval between sections, five to six sections from each rat; Supplementary Table 2). Quantitative analysis of double-labeled OT/cFos neurons was performed offline from all $Z$-stacks from the threehypothalamic nuclei using FLUOVIEW software (Version 3.0, Olympus) by experimenters, who were blind to the treatments during image acquisition as well as during cell counting. For each $Z$-stack image, the cells colocalizing cFos and OT were counted and compared with the 

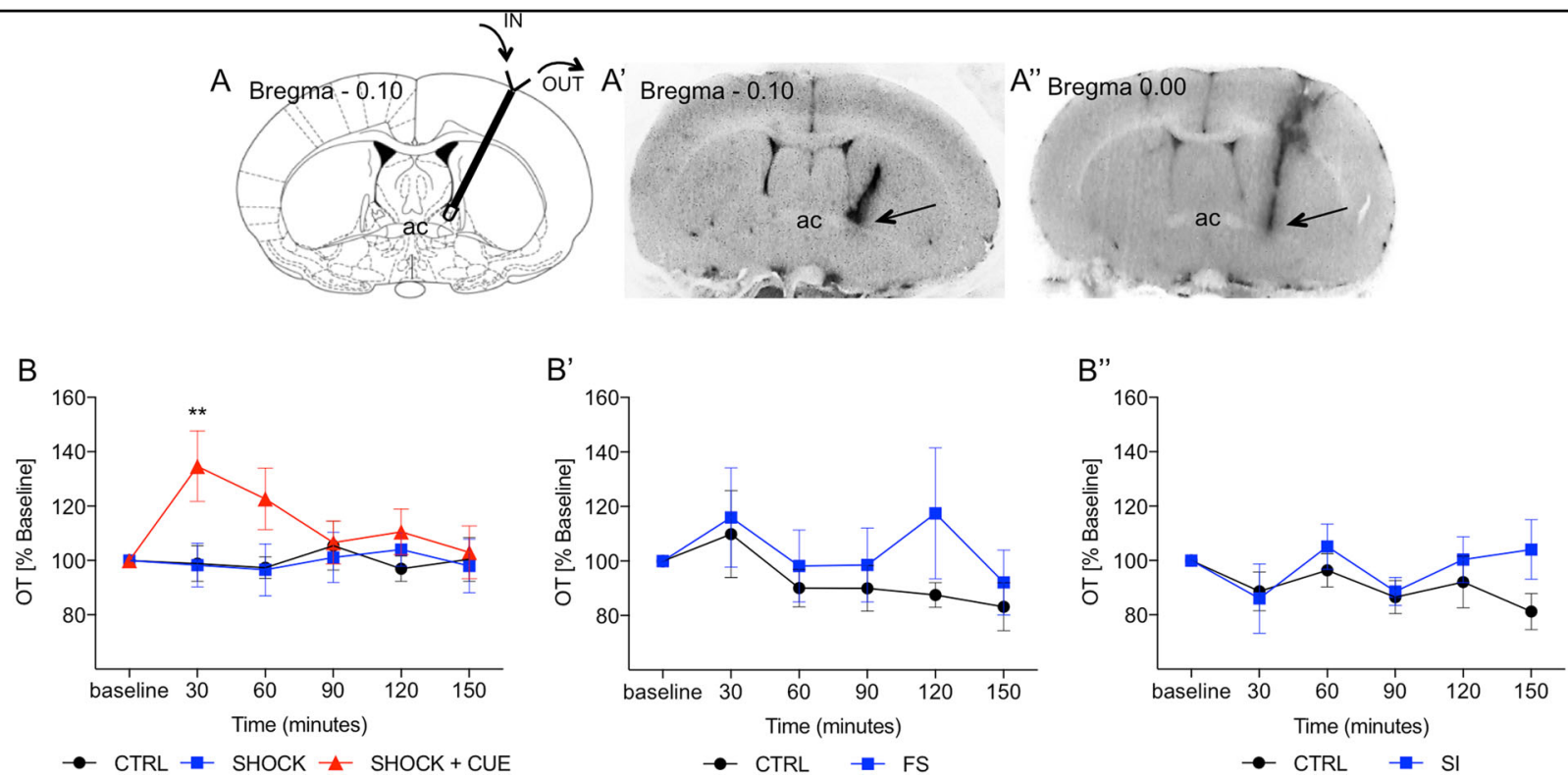

Fig. 2 Fear conditioning, but not acute stress, evokes OT release in the BNST (a, $\left.\mathbf{b}^{\prime \prime}\right)$. Cued fear conditioning evokes OT release in the BNST dl. A-

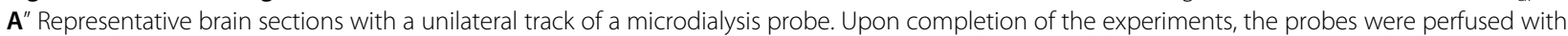
Chicago Sky Blue 6B dye. All extracted brains were sliced and all BNST sections were photographed to confirm proper placement of the probe. Examples of confirmed probe locations in the BNST ${ }_{d l}$ (Bregma $+0.10 \mathrm{~mm}$ to $-0.36 \mathrm{~mm}$ ), which met the following criteria: above the anterior commissure (ac), below the lateral ventricle, and medially to the internal capsule as indicated by the arrows (included in the analysis: $\mathbf{A}^{\prime}$ unilateral probe hit). Examples of misplaced probe locations with probe track too lateral to the BNST dl as indicated by the arrow ( $\mathbf{A}^{\prime \prime}$, excluded from the analysis). B-B" Exposure to foot shocks signaled by a cue, but not unsignaled foot shocks, increases OT content in BNST dl microdialysates. Two-way repeated-measures ANOVA revealed a significant interaction between TIME and TREATMENT $(P=0.0408)$, and post-hoc analysis with Bonferroni's showed a significantly greater percentage change of OT content in BNST dl microdialysates in rats exposed to cue-signaled foot shocks $(134.66 \% \pm$ 12.95 of baseline content) at $30 \mathrm{~min}$ in comparison with CTRL rats $\left(98.86 \% \pm 6.56,{ }^{*} P<0.01\right)$ as well as in comparison with rats exposed to unsignaled foot shocks $\left(98.29 \% \pm 8.04,{ }^{* *} P<0.01\right)(\mathbf{b})$. In contrast, forced swim stress $\left(F S ; \mathbf{B}^{\prime}\right)$ or social interactions $\left(\mathrm{Sl} ; \mathbf{B}^{\prime \prime}\right)$ did not affect OT content in BNST dl microdialysates

total number of neurons expressing OT alone (Supplementary Methods 2.2).

\section{Retrograde tracing of BNST-projecting hypothalamic OT neurons}

Six rats were bilaterally injected into the $\mathrm{BNST}_{\mathrm{dl}}$ with $200 \mathrm{nl}(25 \mathrm{nl} / \mathrm{min})$ of retrograde tracer, cholera toxin B Alexa Fluor 488 conjugate (CTB, Life Technologies, C34775, Eugene, OR). Rats were transcardially perfused 4 weeks later and hypothalamic sections were immunolabeled for OT as above. Imaging was completed using Nikon A1R and Olympus FV10i confocal microscopes.

\section{The effects of OT or OTA administration into the BNST ${ }_{d l}$ on the FPS}

Guide cannulas were implanted bilaterally into the $\mathrm{BNST}_{\mathrm{dl}}$ of 75 rats as before ${ }^{26}$. Twenty-three artificial cerebrospinal fluid (ACSF)-treated, 14-OT, and 16-OTR antagonist (OTA)-treated rats were included in the main analysis. Ten OT- and 12 OTA-treated rats were excluded from the analysis due to cannula misplacement and inability to confirm the location of the cannula (see inclusion criteria in Supplementary Materials 1.4) and were analyzed as negative controls (Fig. 5A-A").

\section{Drugs}

OT acetate salt (H-2510, Bachem, Inc., CA) and a selective OTA (V-905, NIMH Chemical Synthesis and Drug Supply Program) $\left(\mathrm{d}\left(\mathrm{CH}_{2}\right)_{5}{ }^{1}, \mathrm{D}-\mathrm{Tyr}^{2}, \mathrm{Thr}^{4}, \mathrm{Orn}^{8}\right.$, des-Gly- $\left.\mathrm{NH}_{2}{ }^{9}\right)$-vasotocin trifluoroacetate salt ${ }^{36}$ were stored in $-80^{\circ} \mathrm{C}$ freezer and diluted in sterile ACSF $(\mathrm{pH}=7.4)$ before each experiment (Supplementary Methods 3.2).

\section{Fear conditioning and fear recall testing using FPS}

FPS procedures were performed as before ${ }^{10-12,37}$ (Supplementary Methods 3.3). On days 1 (startle habituation) and 2 (pre-test), rats underwent two acoustic startle response (ASR) sessions, they were fear conditioned in context A on day 3 , and $24 \mathrm{~h}$ later rats were tested for cued and non-cued fear expression (recall test, day 4) in context B. On day 5 , the same rats were tested for contextual fear recall in context A (Fig. 1c). 


\section{Data analysis}

FPS analysis, including percentage change of cued and non-cued fear, was performed as before ${ }^{12}$ (Supplementary Methods 3.4). The following formulas were used: Cued fear $=[$ (light-noise trials - noise-alone trials $) /$ noise-alone trials $] \times 100 \%$ in context B. Non-cued fear $=[$ (noise-alone trials - pre-test trials)/pre-test trials $] \times 100 \%$ in context B. Contextual fear $=[$ (noise-alone trials - pre-test trials $) /$ pre-test trials] $\times 100 \%$ in context $\mathrm{A}$. In addition, to determine the ability to discriminate between cued and non-cued fear, we calculated the discrimination index (DI) of individual rats by dividing their percent change score of cued fear by their percent change score of noncued fear responses according to the formula: $\mathrm{DI}=$ [(light-noise trials/noise-alone trials)/(noise-alone trials/ pre-test trials)] in context B.

\section{Statistical analysis}

Data are presented as mean \pm SEM. In the microdialysis experiments, results $(\mathrm{pg} / 100 \mu \mathrm{l})$ were analyzed by a within-group, one-way repeated-measures (RM) analysis of variance (ANOVA) (Supplementary Table 1). For analysis between treatment groups, data are presented as percentage change $( \pm$ SEM) from the subjects' own baseline values and analyzed by a two-way RM ANOVA with the factors TIME and TREATMENT. ANOVA was used to compare percentages of OT neurons colocalizing cFos in each of the hypothalamic nuclei between three conditions (TREATMENT; Supplementary Table 2). FPS data were analyzed by a two-way RM ANOVA with the factors TRIAL TYPE (pre-test, noise-alone, light-noise) and TREATMENT. The percent change scores (cued, noncued, and contextual fear), shock reactivity, and DI were analyzed using ANOVA. To determine the effect of treatment on DI scores as a function of time, results were analyzed with a two-way RM ANOVA with the factors TIME (recall session divided into four blocks, each consisting of five noise-alone and five light-noise trials) and TREATMENT. Where the F-ratio was significant, all pairwise post-hoc comparisons were made using Bonferroni's test. All statistical analyses were completed using GraphPad Prism version 6 (GraphPad Software, Inc., San Diego, CA). $P<0.05$ was considered significant.

\section{Results}

The effects of behavioral manipulations on OT content in BNST $_{\text {dl }}$ microdialysates

OT content $(\mathrm{pg} / 100 \mu \mathrm{l})$ in $\mathrm{BNST}_{\mathrm{dl}}$ microdialysates is shown in Supplementary Table 1.

\section{Cued fear conditioning increases OT content in BNST $T_{d l}$ microdialysates}

To determine whether OT levels were stable before introducing fear conditioning, we performed RM
ANOVA of OT content in three baseline samples and have found stable OT levels in rats exposed to foot shocks alone $(\mathrm{F}(1.150,5.751)=1.977, P=0.2148)$, foot shocks signaled by a cue $(\mathrm{F}(1.502,10.51)=1.740, P=0.2216)$, and control $(\mathrm{CTRL})$ conditions $(\mathrm{F}(1.106,6.636)=1.331, P=$ 0.2948).

To determine whether fear conditioning affected OT content in $\mathrm{BNST}_{\mathrm{dl}}$ microdialysates within each treatment group, we performed RM ANOVA. This revealed a significant TREATMENT effect on OT content in rats exposed to foot shocks signaled by a cue $(\mathrm{F}(3.010,21.07)$ $=3.621, P=0.0297)$, but not rats exposed to foot shocks alone $(\mathrm{F}(2.597,12.98)=0.1707, P=0.8921)$, or CTRL rats $(\mathrm{F}(2.502,15.01)=0.08814, P=0.9468)$.

A two-way RM ANOVA of percentage change from baseline OT content allowed us to perform comparisons between treatment groups. This analysis showed no significant main effect of TREATMENT $(\mathrm{F}(2,20)=1.937$, $P=0.1702) \quad$ or TIME $\quad(\mathrm{F}(5,100)=1.153, \quad P=0.3378)$. However, there was a significant interaction between TIME and TREATMENT $(\mathrm{F}(10,100)=2.002, P=0.0408)$. Bonferroni's multiple comparison test revealed a significantly greater percentage change of OT content in rats exposed to foot shocks signaled by a cue $(134.66 \% \pm$ $12.95)$ at $30 \mathrm{~min}$ in comparison with CTRL rats $(98.86 \% \pm$ $6.56, P<0.01$ ), or in comparison with rats exposed to foot shocks alone $(98.29 \% \pm 8.04, P<0.01)$. No significant effects were observed at $60,90,120$, and $150 \mathrm{~min}$ after the fear conditioning (Fig. 2b).

\section{FS does not affect OT content in BNST $T_{d l}$ microdialysates}

OT content did not differ between baseline microdialysates in rats exposed to $\mathrm{FS}(\mathrm{F}(1.235,8.644)=1.405, P$ $=0.2780) \quad$ or $\quad$ CTRL rats $(\mathrm{F}(1.542,3.88)=1.014$, $P=0.3680$ ).

No significant effect of TREATMENT was observed in rats exposed to $\mathrm{FS}(\mathrm{F}(2.275,15.92)=0.7365, P=0.5109)$ or CTRL rats $(\mathrm{F}(2.510,22.59)=1.220, P=0.3209$, RM ANOVA).

Comparing percentage change from baseline OT content revealed no significant main effect of TREATMENT $(\mathrm{F}(1,16)=1.010, P=0.3298)$ or $\operatorname{TIME}(\mathrm{F}(5,80)=1.414$, $P=0.2282$ ), and no significant interaction between TIME and TREATMENT $(\mathrm{F}(5,80)=0.4828, P=0.7881$, twoway RM ANOVA; Fig. 2b').

\section{Sls do not affect OT content in BNST $T_{\text {dl }}$ microdialysates}

OT content did not differ between baseline $\mathrm{BNST}_{\mathrm{dl}}$ microdialysates in SI rats $(\mathrm{F}(1.115,5.573)=2.160, P=$ $0.1977)$ or CTRL rats $(\mathrm{F}(1.564,9.383)=1.651, P=0.2403)$.

No significant effect of treatment on OT content was observed in rats exposed to $\mathrm{SI}(\mathrm{F}(2.371,11.85)=1.379$, $P=0.2932) \quad$ or $\quad$ CTRL rats $\quad(\mathrm{F}(2.260,15.82)=1.332$, $P=0.2949$ ). 
Comparing percentage changes from baseline OT content revealed no significant main effect of TREATMENT $(\mathrm{F}(1,12)=1.432, \quad P=0.2546)$, no significant effect of TIME $(\mathrm{F}(5,60)=1.394, P=0.2396)$, and no significant interaction between TIME and TREATMENT $(\mathrm{F}(5,60)=$ $0.8411, P=0.5259$; Fig. 2b")

\section{The effect of fear conditioning on OT neurons' activation in the hypothalamus}

Results are shown in Fig. 3. Additional analysis is included in the Supplementary Table 2. The average number of counted OT neurons, including anterior to posterior hypothalamic sections scored bilaterally, was $44.18 \pm 3.17$ for each hemisphere PVN, $59.62 \pm 2.48$ for each SON, and $13.62 \pm 0.80$ for each $\mathrm{AN}$, for all treatment groups combined.

\section{Fear conditioning activates OT neurons in the PVN}

When comparing percentages of cFos-positive OT neurons in the PVN, ANOVA revealed a significant main effect of TREATMENT in response to fear conditioning $(\mathrm{F}(2,129)=3.142, P=0.0465)$. Post-hoc analysis showed a significantly greater percentage of activated OT neurons in rats exposed to foot shocks alone in comparison with CTRL rats $(P=0.0406)$. However, percentage of activated OT neurons did not differ between rats exposed to foot shocks signaled by a cue compared with CTRL rats $(P=$ 0.6495), or compared with rats exposed to foot shocks alone $(P=0.7511)$ (Fig. 3A-A").

\section{Fear conditioning causes robust activation of OT neurons in the SON}

Similarly, in the SON, there was a significant effect of TREATMENT on percentage of activated OT neurons in response to fear conditioning $(\mathrm{F}(2,82)=31.40, \quad P<$ 0.0001 ). Post-hoc analysis revealed a significantly greater percentage of activated OT neurons in rats exposed to foot shocks signaled by a cue $(P=0.0030)$ and foot shocks alone $(P<0.0001)$ in comparison with CTRL rats. A significant difference was also observed in rats exposed to signaled vs. unsignaled footshocks $(P=0.0003)$ (Fig. 3B-B").

\section{Fear conditioning causes robust activation of OT neurons in the AN}

A significant TREATMENT effect $(\mathrm{F}(2,150)=12.62$, $P<0.0001)$ followed by a post-hoc analysis revealed a significantly greater percentage of activated OT neurons in rats exposed to foot shocks signaled by a cue $(P=$ $0.0168)$ as well as group exposed to foot shocks alone $(P<$ 0.0001), as compared with CTRL. Percentages of activated OT neurons did not differ between the two groups $(P=$ 0.2564) (Fig. 3C-C"). Interestingly, the most intense OTcFOS co-expression was observed in posterior AN sections in both groups, reaching $25 \%$ of all OT neurons co-expressing cFos (Bregma -1.80 to -2.28 based on Rat Brain Atlas ${ }^{38}$; Supplementary Table 2.

\section{Exposure to cue alone does not activate OT neurons in the hypothalamus}

To determine whether exposure to CS alone can cause an activation of OT neurons, percentage of activated OT neurons was compared between rats exposed to cue (light) alone and control rats. In the PVN, the percentage of OT neurons co-expressing cFos did not differ between rats exposed to cue alone and control rats $(t=0.5609$, $\mathrm{df}=45, P=0.5776$, unpaired $t$-test). Similarly, in the SON, no treatment effect was detected $(t=0.7189$, $\mathrm{df}=27, P=0.4784)$. Finally, no cue effect was also observed in the AN $(t=1.063, \mathrm{df}=32, P=0.2959)$.

OT neurons from the hypothalamus project to the BNST $\mathrm{dl}_{\mathrm{dl}}$

To determine which hypothalamic OT neurons project to the $\mathrm{BNST}_{\mathrm{dl}}$, we injected a retrograde tracer, CTB, into the $\mathrm{BNST}_{\mathrm{dl}}$, and several weeks later we stained serial hypothalamic brain sections with OT. Double-labeled neurons for CTB-Alexa 488 and OT were found in the PVN (Fig. 4A-A"), anterior SON (Fig. 4B-B", Bregma -1.20 to Bregma $-1.32 \mathrm{~mm}$ ), and posterior $\mathrm{AN}$ (Fig. 4C-C"), indicative of BNST-projecting OT neurons.

\section{Effects of OT or OTA administration into the BNST ${ }_{d l}$ on fear acquisition \\ Acquisition of cued fear conditioning}

All animals exhibited a significantly potentiated startle in light-noise trials compared with noise-alone trials. Two-way RM ANOVA showed a significant main effect of TRIAL TYPE $(\mathrm{F}(1,50)=32.01, P<0.0001)$ but no main effect of TREATMENT $(\mathrm{F}(2,50)=0.1656, P=0.8478)$ and no interaction between TRIAL TYPE and TREATMENT $(F(2,50)=0.7115, P=0.4958$; Fig. 5b). Comparison of percentage changes revealed a trend in the TREATMENT effect on cued fear $(\mathrm{F}(2,50)=2.433, P=$ 0.0981, ANOVA; Fig. 5c). As high variability was observed in OT-treated rats, we also compared ACSF and OTAtreated groups using unpaired $t$-test, which revealed a trend in the OTA effect on cued fear $(P=0.0763)$.

\section{Acquisition of non-cued fear conditioning}

Quantitative analysis showed a significant enhancement of ASR in noise-alone trials compared with pre-test trials across all groups. There was a main effect of TRIAL TYPE (pre-test vs. noise-alone, $\mathrm{F}(1,50)=22.73, P<0.0001$ ) but no main effect of TREATMENT $(\mathrm{F}(2,50)=0.8546, P=$ $0.4316)$ and no significant interaction $(\mathrm{F}(2,50)=0.6216$, $P=0.5412$ ). When comparing percentage change of noncued fear with ANOVA, no significant differences were 


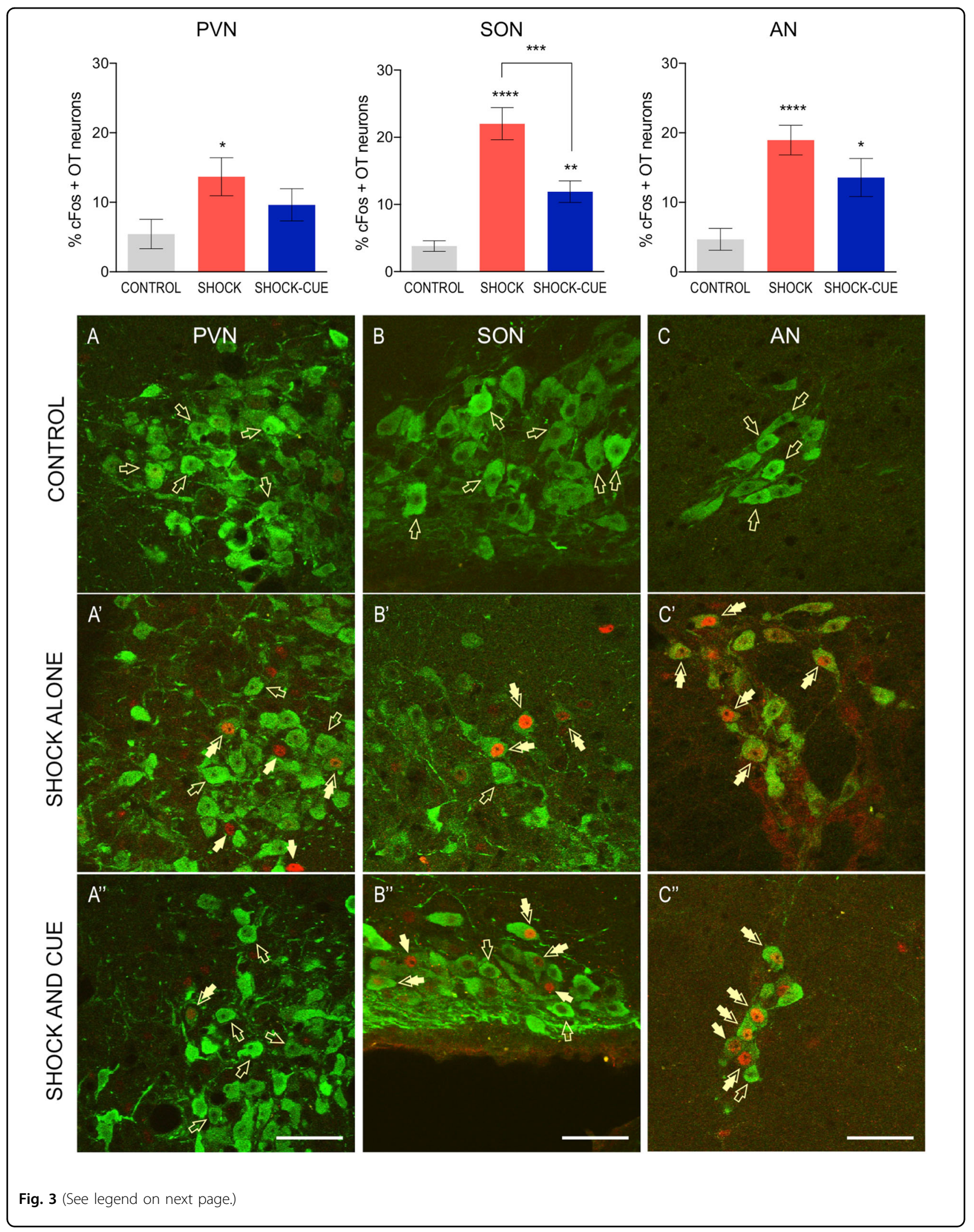


Fig. 3 Fear conditioning increases percentage of activated OT neurons in the PVN, SON, and AN. Upper panel: in the PVN, there was a significant effect of fear conditioning on OT neurons activation $(P=0.0465)$, with a significantly greater percentage of activated OT neurons in rats exposed to foot shocks alone in comparison with control rats $\left({ }^{*} P<0.05\right)$. OT neurons within the SON were significantly activated in response to fear conditioning $(P<0.0001$, one-way ANOVA), with a greater percentage of OT neurons co-expressing cFos in rats exposed to un-signaled foot shocks compared with control rats $\left({ }^{* * *} P<0.0001\right)$, as well as in rats exposed to foot shocks signaled by a cue compared with controls $\left({ }^{* *} P<0.01\right)$. Finally, there was a greater activation of OT neurons in response to foot shocks alone vs. foot shocks signaled by a cue in the SON $(* * * P<0.001)$. Fear conditioning activated OT neurons in the AN ( ${ }^{* * *} P<0.0001$, one-way ANOVA), with a significantly greater percentage of activated OT neurons in rats exposed to unsignaled foot shocks $\left({ }^{* * *} P<0.0001\right)$ and in rats exposed to foot shocks signaled by a cue $\left({ }^{*} P=0.0168\right)$, compared with control rats. Bottom panel: although control rats show little co-expression of OT (green, open arrows) and cFos (red, closed arrows) in the PVN (A), rats exposed to foot shocks alone $\left(\mathbf{A}^{\prime}\right)$, but not foot shocks signaled by a cue $\left(\mathbf{A}^{\prime \prime}\right)$, show increase in percentage of OT neurons co-expressing CFos in the PVN. In the SON, number of OT neurons co-expressing CFos was significantly increased in response to unsignaled foot shocks (B') as well as foot shocks signaled by a cue $\left(\mathbf{B}^{\prime \prime}\right)$, in comparison with control rats (B). In the AN, percentage of OT neurons co-expressing cFos was increased in response to foot shocks alone $\left(\mathbf{C}^{\prime}\right)$ as well as foot shocks signaled by a cue $\left(\mathbf{C}^{\prime \prime}\right)$, in comparison with control rats ( $\mathbf{C}^{\prime \prime}$, magnification $\times 60$, scale bar $\left.50 \mu \mathrm{m}\right)$

observed between treatment groups $(\mathrm{F}(2,50)=0.1063$, $P=0.8993$; Fig. 5d).

\section{Acquisition of contextual fear conditioning}

Quantitative analysis showed a significant enhancement of ASR in the training context compared with pre-test trials. There was a main effect of TRIAL TYPE $(F(1,46)=$ $12.45, P=0.001$ ) but no main effect of TREATMENT (F $(2,6)=1.35, P=0.2693)$ and no significant interaction ( $\mathrm{F}$ $(2,46)=1.096, P=0.3429)$. Similarly, the mean percent change analysis showed that contextual fear did not differ between treatment groups $(\mathrm{F}(2,46)=0.6275, P=0.5384$; Fig. 5e).

\section{Shock reactivity}

The mean shock reactivity during the fear-conditioning session was not different between treatment groups $(\mathrm{F}(2,50)=0.1415, P=0.8684$; Fig. $5 \mathrm{f})$.

\section{Discrimination index}

The calculated overall DI was not significantly different between treatment groups $(\mathrm{F}(2,50)=1.977, P=0.1492$, ANOVA; Fig. 5g). When the DI was calculated over four time blocks, there was no significant main effect of TREATMENT $\quad(\mathrm{F}(2,50)=1.92, \quad P=0.1573) \quad$ or $\quad$ TIME $(\mathrm{F}(3,150)=1.47, P=0.2249)$, but there was a significant interaction between TREATMENT and TIME $(\mathrm{F}(6,150)=$ $2.261, P=0.0406$, two-way RM ANOVA). Post-hoc comparisons revealed significant differences in the DI in the fourth time block of fear memory recall between ACSFand OT-treated groups $(t(200)=2.91, P=0.0121)$, as well as between OT and OTA-treated groups $(t(200)=3.739$, $P=0.0007$, Bonferroni's test; Fig. 5h). In the negative controls, the DI over four time blocks revealed no significant main effect of TREATMENT $(\mathrm{F}(2,42)=0.5469$, $P=0.5828)$ or TIME $(\mathrm{F}(3,126)=0.6473, P=0.5861)$ and there was no significant interaction $(F(6,126)=0.2376$, $P=0.9634$; Fig. 5h').

\section{Discussion}

In the current study, using in vivo microdialysis in freely moving male rats as well as brain-site-specific in vivo pharmacology, we demonstrate that OTR transmission in the $\mathrm{BNST}_{\mathrm{dl}}$ enables fear learning of cued (signaled, predictable) fear. Using double immunofluorescence labeling of OT and immediate early gene expression cFos, we show that in the hypothalamus, exposure to fear conditioning causes robust activation of OT neurons in the SON and posterior AN.

We previously demonstrated that intra- $\mathrm{BNST}_{\mathrm{dl}}$ infusion of an OTR antagonist before fear conditioning significantly reduces cued fear recall ${ }^{12}$. In the current study we show that presentation of foot shocks signaled by a discrete cue leads to a significant increase in OT content in $\mathrm{BNST}_{\mathrm{dl}}$ microdialysates, whereas the presentation of unsignaled foot shocks has no effect. In contrast to previous studies in the $\mathrm{PVN}^{39,40}$, central amygdala ${ }^{41}$, and lateral septum ${ }^{42}$, we demonstrate that OT release in the $\mathrm{BNST}_{\mathrm{dl}}$ is not sensitive to acute stress of FS or foot shocks alone. However, caution needs to be applied when interpreting forced swim results, as high variability of OT content in the $\mathrm{BNST}_{\mathrm{dl}}$ microdialysates was observed in this experimental group. Previous studies have shown that OT content in the posterior BNST correlate with social discrimination in rats ${ }^{43}$. Although we did not specifically employ a social recognition paradigm, we demonstrate that OT release in the $\mathrm{BNST}_{\mathrm{dl}}$ is not modulated by SIs with a novel rat. Our results suggest that $\mathrm{OT}$ in the $\mathrm{BNST}_{\mathrm{dl}}$ is released during the acquisition of cued fear, and that OT neurons in the hypothalamus are activated in response to fear conditioning.

Accordingly, we show a significant increase in the percentage of activated OT neurons in the SON and AN in response to signaled and unsignaled foot shocks. Interestingly, the AN has been identified as the main source of OT innervation in the $\mathrm{CeA}^{7}$, a critical brain region for acquisition and consolidation of fear memory ${ }^{44}$. We also show activation of OT neurons in the PVN in rats exposed to foot shocks alone, but not cue-signaled foot 


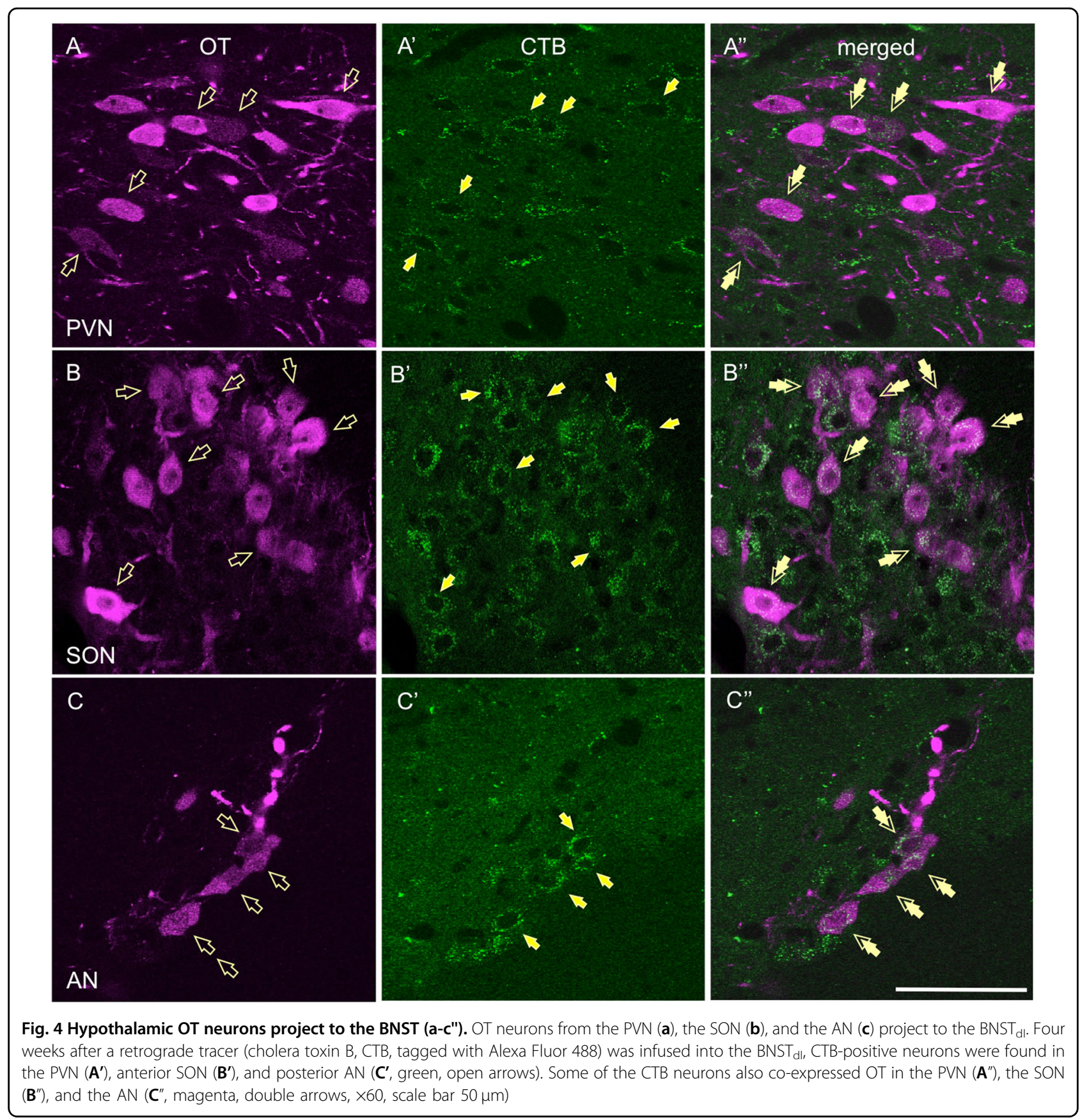

shocks. Similarly, acute stressors were shown to activate OT neurons in the $\mathrm{PVN}^{45,46}$. However, although the PVN sends OT projections to the $\mathrm{BNST}_{\mathrm{dl}}{ }^{7,30}$, we show that $\mathrm{OT}$ release in the $\mathrm{BNST}_{\mathrm{dl}}$ is not evoked by unsignaled foot shocks. Although seemingly contradictory, another population of BNST-projecting hypothalamic OT neurons might modulate acquisition of cued fear, as it has been shown that discrete clusters of OT neurons are highly specialized in their projections and functions ${ }^{47}$. Using retrograde neuronal tracing, we demonstrate that
OT neurons from anterior SON and posterior $\mathrm{AN}$ also send projections to the $\mathrm{BNST}_{\mathrm{dl}}$, suggesting they might be specifically involved in facilitating cued fear. Our future studies will identify specific populations of OT neurons in the SON and AN activated by cue-signaled foot shocks. Previously, recall of cued fear conditioning or recall of foot shock alone has been shown to activate OT neurons in the SON and $\mathrm{PVN}^{48}$. Notably, in our study, exposure to foot shocks alone generally activated more OT neurons in the hypothalamic nuclei, suggesting that cue presentation 


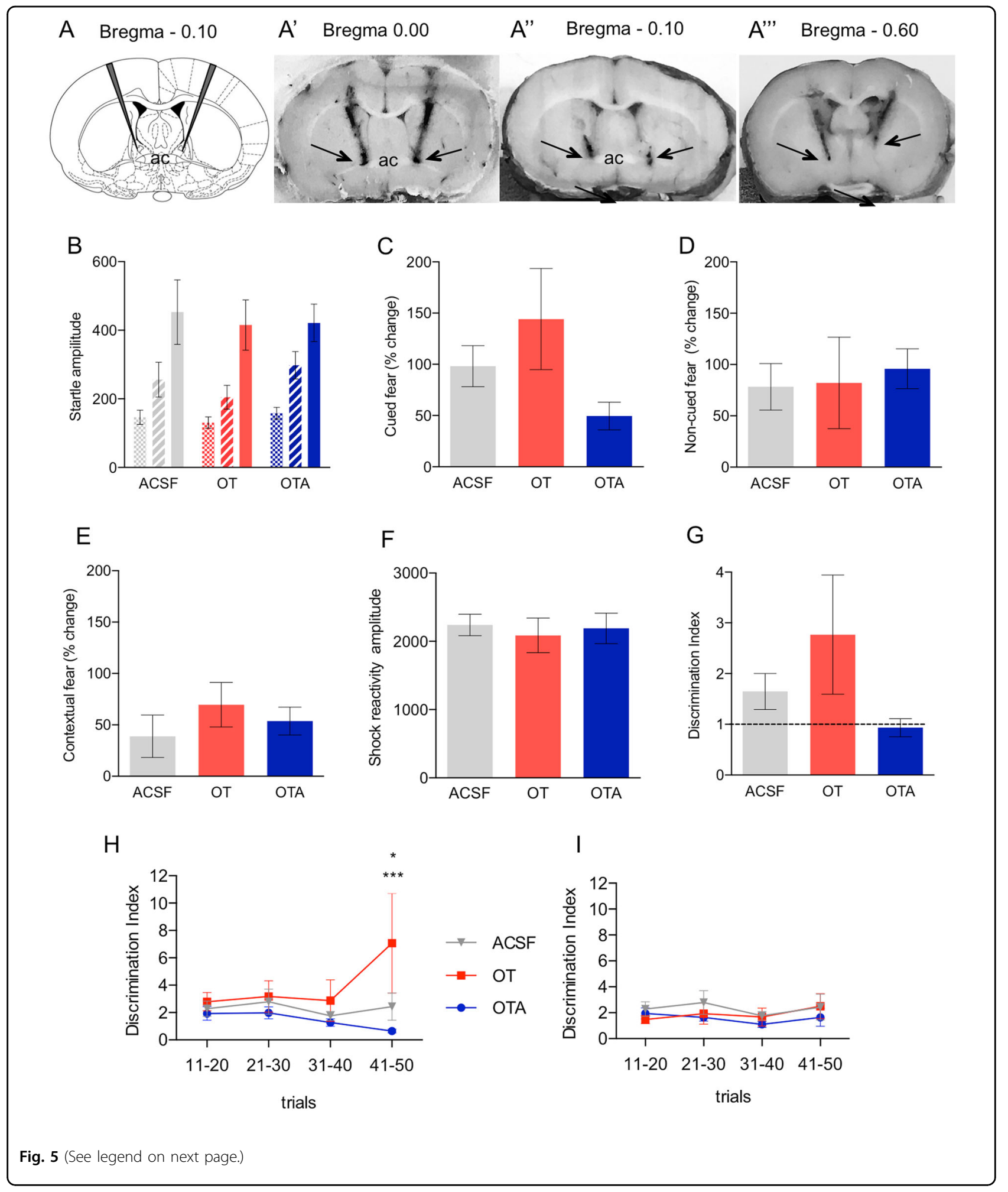

may have suppressed the activation of OT neurons. However, our control experiment shows that cue alone does not affect OT neurons' activation in any of the hypothalamic nuclei. It is therefore possible that OT projection to the $\mathrm{BNST}_{\mathrm{dl}}$ is selectively involved in strengthening formation of cued fear. Once activated by OT, BNST neurons might have inhibited hypothalamic OT neurons, leading to the observed reduction of their 


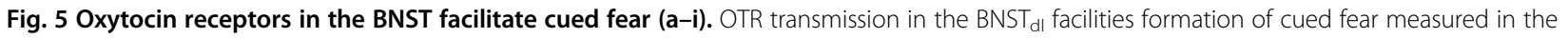
FPS. A-A $\mathbf{A}^{\prime \prime \prime}$ Representative brain sections with bilateral cannulas targeting the BNST d. Bilateral cannula hit $\left(\mathbf{A}^{\prime}\right)$, unilateral cannula hit $\left(\mathbf{A}^{\prime \prime}\right)$, or cannula too posterior to the BNST $T_{d l}\left(\mathbf{A}^{\prime \prime \prime}\right)$. $\mathbf{b}-\mathbf{g}$ The effects of intra-BNST $T_{d l}$ administration of ACSF, OT, or OTA on acquisition of fear conditioning measured in the FPS. Group data for pre-test, noise-alone, and light-noise startle amplitude from rats given bilateral intra-BNST $d 1$ ACSF ( $n=23$, gray), OT (100 ng, $n$ $=14$, red), or OTA (200 $\mathrm{ng}, n=16$, blue), 10 min before the fear-conditioning session. All rats exhibited a significantly potentiated startle response in light-noise trials compared with noise-alone trials $(P<0.0001)$, but this was not affected by the treatment $(\mathbf{b})$. There was a trend toward TREATMENT effect on the percentage change of cued fear in rats given intra-BNST dl ACSF, OT, or OTA $(P=0.0981)$ (c). All rats exhibited a significant potentiation of startle amplitude in noise-alone trials in comparison with pre-test ASR $(P<0.0001)$, but it was not affected by intra-BNST d $_{\text {injections }}(\mathbf{b})$. There was no TREATMENT effect on percentage change on non-cued fear $(P=0.8993)(\mathbf{d})$, contextual fear $(P=0.5384(\mathbf{e})$, or shock reactivity $(P=0.8684)(\mathbf{f})$. Comparing discrimination indices from all trials in rats injected with ACSF, OT, and OTA did not show any significant effect of TREATMENT $(P=$

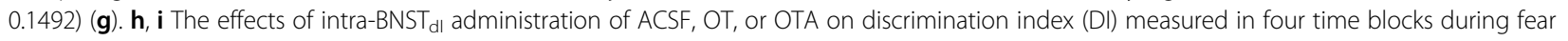
memory recall. Each block consists of five noise-alone trials and five light-noise trials, which have been used to calculate DI in each block. There was a significant interaction between TIME and TREATMENT $(P=0.0406)$ and Bonferroni's post-hoc tests showed a significant difference in the fourth time block between DI of rats injected with ACSF and OT $(P=0.0121)$ as well as rats injected with OT and OTA $\left(P=0.0007,{ }^{* * *} P<0.001,{ }^{*} P<0.05\right)(\mathbf{h})$. DI calculated over four time blocks during fear memory recall in negative controls (injections sites outside the BNST ${ }_{\text {dll }}$ ) showed no main effect of TREATMENT $(P=0.5828)$ nor an interaction between TIME and TREATMENT $(P=0.9634)(\mathbf{i})$. Cued fear $=[($ light-noise trials - noise-alone trials $) /$ noisealone trials $] \times 100 \%$ in context B. Non-cued fear $=[($ noise-alone trials - pre-test trials $) /$ pre-test trials $] \times 100 \%$ in context B. Contextual fear $=[($ noisealone trials - pre-test trials)/pre-test trials] $\times 100 \%$ in context $\mathrm{A}$

activation. In fact, projection from GABA-ergic neurons in the $\mathrm{BNST}_{\mathrm{dl}}$ to OT neurons in the PVN has been shown before ${ }^{49}$.

Although we demonstrated that exposure to cue alone does not induce an activation of OT neurons, we could not perform a microdialysis experiment during an exposure to cue alone. Here, microdialysates' sampling was further complicated by the fact that, once placed in SRLAB cylindrical enclosures, and not distracted by foot shocks exposure, freely moving rats quickly engage in the consumption and damage of the microdialysis tubing. Nonetheless, as cue alone does not induce any activation of OT neurons, it is unlikely to be that it would induce OT release in the $\mathrm{BNST}_{\mathrm{dl}}$.

We next investigated the role of OTR transmission in the $\mathrm{BNST}_{\mathrm{dl}}$ in the acquisition of cued, non-cued, and contextual fear. Structural modification in the OTR antagonist (D-Tyr ${ }^{2}$ instead of $\operatorname{Tyr}(\mathrm{Me})^{2}$ ) renders this compound more selective toward OTR vs. vasopressin receptors in comparison with the antagonist we used before $^{12}$ (also see ref. ${ }^{36}$ ). However, in contrast to our previous findings, we show that blocking OTR in the BNST $_{\mathrm{dl}}$ before fear conditioning does not significantly reduce cued fear but only induces a trend in cued fear reduction measured in the FPS. However, by calculating DI, we show that infusion of OT potentiates fear discrimination and strengthens fear responses toward cued fear, especially during the later phase of fear recall. Nonetheless, OT-treated group shows high variability of fear responses. Considering that endogenous OT is released during fear acquisition, infusing more exogenous OT might have led to high variability of fear responses. Using selective OTR agonist, $\left(\mathrm{Thr}^{4}, \mathrm{Gly}^{7}\right)$-OT, might be a better alternative in the future studies. In previous FPS studies, systemic, but not ICV, administration of OT reduced non-cued fear (background anxiety), without affecting cued or contextual fear ${ }^{10,11}$. Therefore, it is possible that OT dynamically modulates both cued and non-cued fear responses in an opposite manner; it facilitates cued fear in the BNST and reduces non-cued fear responses, overall promoting discrimination between the two. In the $\mathrm{BNST}_{\mathrm{dl}}$, OT might activate neurons mediating cued fear, which in turn might inhibit neurons responsible for non-cued, sustained fear responses in, or outside the $\mathrm{BNST}_{\mathrm{dl}}\left(\right.$ see ref. ${ }^{13}$ ). Such dualism was reported in the BNST after auditory fear conditioning, where anterolateral neurons were inhibited, whereas anteromedial BNST neurons were excited in response to $\mathrm{CS}^{+}$presentation ${ }^{50}$. Notably, a similar role of OTR in facilitating fear discrimination has been recently shown in the basolateral amygdala ${ }^{51}$.

Our findings have translational validity for psychiatric disorders in humans. Although healthy controls have greater startle responses to stimuli predicting danger vs. stimuli predicting safety, humans suffering from PTSD demontrate lack of dicrimination between these stimuli ${ }^{52}$. However, startle responses to aversive stimuli adiministered in a predictable manner do not differ between PTSD patients and healthy controls ${ }^{53}$. This highlights the adaptive role of cued/predictable fear, which is necessary to accurately detect and avoid danger. The notion of OT strengthening the adaptive fear and improving the ability to accurately discriminate between danger and safety becomes apparent in animal and human research ${ }^{13,54}$. Notably, a growing number of studies emphasize the role of the BNST in the discrimination between cued (signaled, predictable, phasic) vs. non-cued (unsignaled, unpredictable, sustained) fear ${ }^{13,25-29,55}$. OTR transmission in the $\mathrm{BNST}_{\mathrm{dl}}$ might play a pivotal role in learning to accurately recognize threats signaled by a dicrete cue. 


\section{Acknowledgements}

This work was supported by grant from the National Institute of Mental Health R01 MH113007 to J.D., DePaul-RFUMS seed research grant to J.D., as well as start-up funds from the Chicago Medical School, Rosalind Franklin University of Medicine and Science to J.D. Dr Joanna Dabrowska reports submission of a provisional patent application entitled Method and System for Testing for Stress-related Mental Disorders, Susceptibility, Disease Progression, Disease Modulation and Treatment Efficacy (\# 62/673447).

\section{Author details}

${ }^{1}$ Department of Cellular and Molecular Pharmacology, Chicago Medical School, Rosalind Franklin University of Medicine and Science, North Chicago, IL 60064, USA. ${ }^{2}$ Center for the Neurobiology of Stress Resilience and Psychiatric Disorders, Rosalind Franklin University of Medicine and Science, North Chicago, IL 60064, USA. ${ }^{3}$ School of Graduate and Postdoctoral Studies, Rosalind Franklin University of Medicine and Science, North Chicago, IL 60064, USA

\section{Author contributions}

J.D. designed the study. D.M., P.L., A.N.R., P.T., S.V.A., G.B., and V.O. acquired data. D.M., P.L., A.N.R., P.T., S.V.A., V.O., and J.D. analyzed and interpreted data. J.D. wrote the manuscript. P.L., A.N.R., P.T., and S.V.A. edited the manuscript.

\section{Conflict of interest}

The authors declare that they have no conflict of interest.

\section{Publisher's note}

Springer Nature remains neutral with regard to jurisdictional claims in published maps and institutional affiliations.

Supplementary Information accompanies this paper at (https://doi.org/ 10.1038/s41398-019-0474-x).

Received: 21 November 2018 Revised: 19 March 2019 Accepted: 1 April 2019

Published online: 18 April 2019

\section{References}

1. Du Vigneaud, V. Trail of sulfur research: from insulin to oxytocin. Science (New York, NY) 123, 967-974 (1956).

2. Jurek, B. \& Neumann, I. D. The oxytocin receptor: from intracellular signaling to behavior. Physiol. Rev. 98, 1805-1908 (2018).

3. Neumann, I. D. \& Slattery, D. A. Oxytocin in general anxiety and social fear: a translational approach. Biol. Psychiatry 79, 213-221 (2016).

4. Bale, T. L., Davis, A. M., Auger, A. P., Dorsa, D. M. \& McCarthy, M. M. CNS regionspecific oxytocin receptor expression: importance in regulation of anxiety and sex behavior. J. Neurosci. 21, 2546-2552 (2001).

5. Ellenbogen, M. A., Linnen, A. M., Cardoso, C. \& Joober, R. Intranasal oxytocin attenuates the human acoustic startle response independent of emotional modulation. Psychophysiology 51, 1169-1177 (2014).

6. Ring, R. H. et al. Anxiolytic-like activity of oxytocin in male mice: behavioral and autonomic evidence, therapeutic implications. Psychopharmacology $\mathbf{1 8 5}$ 218-225 (2006).

7. Knobloch, H. S. et al. Evoked axonal oxytocin release in the central amygdala attenuates fear response. Neuron 73, 553-566 (2012).

8. Lahoud, N. \& Maroun, M. Oxytocinergic manipulations in corticolimbic circuit differentially affect fear acquisition and extinction. Psychoneuroendocrinology 38, 2184-2195 (2013).

9. Guzman, Y. F. et al. Fear-enhancing effects of septal oxytocin receptors. Nat. Neurosci. 16, 1185-1187 (2013)

10. Ayers, L. W., Missig, G., Schulkin, J. \& Rosen, J. B. Oxytocin reduces background anxiety in a fear-potentiated startle paradigm: peripheral vs central administration. Neuropsychopharmacology 36, 2488-2497 (2011).

11. Missig, G., Ayers, L. W., Schulkin, J. \& Rosen, J. B. Oxytocin reduces background anxiety in a fear-potentiated startle paradigm. Neuropsychopharmacology 35, 2607-2616 (2010).

12. Moaddab, M. \& Dabrowska, J. Oxytocin receptor neurotransmission in the dorsolateral bed nucleus of the stria terminalis facilitates the acquisition of cued fear in the fear-potentiated startle paradigm in rats. Neuropharmacology 121, 130-139 (2017).

13. Janecek, M. \& Dabrowska, J. Oxytocin facilitates adaptive fear and attenuates anxiety responses in animal models and human studies-potential interaction with the corticotropin-releasing factor (CRF) system in the bed nucleus of the stria terminalis (BNST). Cell Tissue Res. 375, 143-172 (2018).

14. Dabrowska, J. et al. Striatal-enriched protein tyrosine phosphatase-STEPs toward understanding chronic stress-induced activation of corticotrophin releasing factor neurons in the rat bed nucleus of the stria terminalis. Biol. psychiatry 74, 817-826 (2013).

15. Daniel, S. E. \& Rainnie, D. G. Stress Modulation of Opposing Circuits in the Bed Nucleus of the Stria Terminalis. Neuropsychopharmacology 41, 103-125 (2016).

16. Davis, M., Walker, D. L., Miles, L. \& Grillon, C. Phasic vs sustained fear in rats and humans: role of the extended amygdala in fear vs anxiety. Neuropsychopharmacology 35, 105-135 (2010).

17. Yassa, M. A., Hazlett, R. L., Stark, C. E. \& Hoehn-Saric, R. Functional MRI of the amygdala and bed nucleus of the stria terminalis during conditions of uncertainty in generalized anxiety disorder. J. Psychiatr. Res. 46, 1045-1052 (2012).

18. Somenille, L. H., Whalen, P. J. \& Kelley, W. M. Human bed nucleus of the stria terminalis indexes hypervigilant threat monitoring. Biol. Psychiatry $\mathbf{6 8}, 416-424$ (2010).

19. Straube, T., Mentzel, H. J. \& Miltner, W. H. Waiting for spiders: brain activation during anticipatory anxiety in spider phobics. Neurolmage 37, 1427-1436 (2007).

20. Sullivan, G. M. et al. Lesions in the bed nucleus of the stria terminalis disrupt corticosterone and freezing responses elicited by a contextual but not by a specific cue-conditioned fear stimulus. Neuroscience 128, 7-14 (2004).

21. Waddell, J., Morris, R. W. \& Bouton, M. E. Effects of bed nucleus of the stria terminalis lesions on conditioned anxiety: aversive conditioning with longduration conditional stimuli and reinstatement of extinguished fear. Behav. Neurosci. 120, 324-336 (2006).

22. Gewirtz, J. C., McNish, K. A. \& Davis, M. Lesions of the bed nucleus of the stria terminalis block sensitization of the acoustic startle reflex produced by repeated stress, but not fear-potentiated startle. Prog. Neuropsychopharmacol. Biol. Psychiatry 22, 625-648 (1998).

23. Hitchcock, J. M. \& Davis, M. Efferent pathway of the amygdala involved in conditioned fear as measured with the fear-potentiated startle paradigm. Behav. Neurosci. 105, 826-842 (1991).

24. LeDoux, J. E., Iwata, J., Cicchetti, P. \& Reis, D. J. Different projections of the central amygdaloid nucleus mediate autonomic and behavioral correlates of conditioned fear. J. Neurosci. 8, 2517-2529 (1988).

25. Goode, T. D. \& Maren, S. Role of the bed nucleus of the stria terminalis in aversive learning and memory. Learn. Mem. 24, 480-491 (2017).

26. Gungor, N. Z. \& Pare, D. Functional heterogeneity in the bed nucleus of the stria terminalis. J. Neurosci. 36, 8038-8049 (2016).

27. Duvarci, S., Bauer, E. P. \& Pare, D. The bed nucleus of the stria terminalis mediates inter-individual variations in anxiety and fear. J. Neurosci. 29, 10357-10361 (2009)

28. De Bundel, D. et al. Dopamine D2 receptors gate generalization of conditioned threat responses through mTORC1 signaling in the extended amygdala. Mol. Psychiatry 21, 1545-1553 (2016).

29. Lange, M. D. et al. Cannabinoid CB1 receptors in distinct circuits of the extended amygdala determine fear responsiveness to unpredictable threat. Mol. Psychiatry 22, 1422-1430 (2017).

30. Dabrowska, J. et al. Neuroanatomical evidence for reciprocal regulation of the corticotrophin-releasing factor and oxytocin systems in the hypothalamus and the bed nucleus of the stria terminalis of the rat: Implications for balancing stress and affect. Psychoneuroendocrinology 36, 1312-1326 (2011).

31. Dumais, K. M., Bredewold, R., Mayer, T. E. \& Veenema, A. H. Sex differences in oxytocin receptor binding in forebrain regions: correlations with social interest in brain region- and sex- specific ways. Horm. Behav. 64, 693-701 (2013).

32. Tribollet, E., Dubois-Dauphin, M., Dreifuss, J. J., Barberis, C. \& Jard, S. Oxytocin receptors in the central nervous system. Distribution, development, and species differences. Ann. N. Y. Acad. Sci. 652, 29-38 (1992).

33. Veinante, P. \& Freund-Mercier, M. J. Distribution of oxytocin- and vasopressinbinding sites in the rat extended amygdala: a histoautoradiographic study. J. Comp. Neurol. 383, 305-325 (1997).

34. Martinon, D. \& Dabrowska, J. Corticotropin-releasing factor receptors modulate oxytocin release in the dorsolateral bed nucleus of the stria terminalis (BNST) in male rats. Front. Neurosci. 12, 183 (2018). 
35. Dabrowska, J., Nowak, P. \& Brus, R. Reactivity of 5-HT1A receptor in adult rats after neonatal noradrenergic neurons' lesion-implications for antidepressantlike action. Brain Res. 1239, 66-76 (2008).

36. Manning, M. et al. Oxytocin and vasopressin agonists and antagonists as research tools and potential therapeutics. J. Neuroendocrinol. 24, 609-628 (2012).

37. Walker, D. et al. Differential effects of the CRF-R1 antagonist GSK876008 on fear-potentiated, light- and CRF-enhanced startle suggest preferential involvement in sustained vs phasic threat responses. Neuropsychopharmacology $\mathbf{3 4}$ 1533-1542 (2009)

38. Paxinos, G. \& Watson, C. The Rat Brain in Stereotaxic Coordinates. 6th ed. (Academic Press, London, 2007).

39. Bosch, O. J., Kromer, S. A., Brunton, P. J. \& Neumann, I. D. Release of oxytocin in the hypothalamic paraventricular nucleus, but not central amygdala or lateral septum in lactating residents and virgin intruders during maternal defence. Neuroscience 124, 439-448 (2004).

40. Neumann, I. D. Stimuli and consequences of dendritic release of oxytocin within the brain. Biochem. Soc. Trans. 35, 1252-1257 (2007).

41. Ebner, K., Bosch, O. J., Kromer, S. A., Singewald, N. \& Neumann, I. D. Release of oxytocin in the rat central amygdala modulates stress-coping behavior and the release of excitatory amino acids. Neuropsychopharmacology 30, 223-230 (2005).

42. Ebner, K., Wotjak, C. T., Landgraf, R. \& Engelmann, M. A single social defeat experience selectively stimulates the release of oxytocin, but not vasopressin, within the septal brain area of male rats. Brain Res. 872, 87-92 (2000).

43. Dumais, K. M., Alonso, A. G., Immormino, M. A., Bredewold, R. \& Veenema, A. H. Involvement of the oxytocin system in the bed nucleus of the stria terminalis in the sex-specific regulation of social recognition. Psychoneuroendocrinology 64, 79-88 (2016).

44. Wilensky, A. E., Schafe, G. E., Kristensen, M. P. \& LeDoux, J. E. Rethinking the fear circuit: the central nucleus of the amygdala is required for the acquisition, consolidation, and expression of Pavlovian fear conditioning. J. Neurosci. 26, 12387-12396 (2006)
45. Robinson, D. A. et al. Oxytocin mediates stress-induced analgesia in adult mice. J. Physiol. 540, 593-606 (2002).

46. Wotjak, C. T. et al. Forced swimming stimulates the expression of vasopressin and oxytocin in magnocellular neurons of the rat hypothalamic paraventricular nucleus. Eur. J. Neurosci. 13, 2273-2281 (2001).

47. Eliava, M. et al. A new population of parvocellular oxytocin neurons controlling magnocellular neuron activity and inflammatory pain processing. Neuron $\mathbf{8 9}$, 1291-1304 (2016)

48. Zhu, L. \& Onaka, T. Involvement of medullary A2 noradrenergic neurons in the activation of oxytocin neurons after conditioned fear stimuli. Eur. J. Neurosci. 16, 2186-2198 (2002)

49. Dabrowska, J., Martinon, D., Moaddab, M. \& Rainnie D. G. Targeting corticotropin-releasing factor (CRF) projections from the oval nucleus of the BNST using cell-type specific neuronal tracing studies in mouse and rat brain. J. Neuroendocrinol. 28 (2016).

50. Haufler, D., Nagy, F. Z. \& Pare, D. Neuronal correlates of fear conditioning in the bed nucleus of the stria terminalis. Learn. Mem. (Cold Spring Harb., NY). 20, 633-641 (2013)

51. Fam, J., Holmes, N., Delaney, A., Crane, J. \& Westbrook, R. F. Oxytocin receptor activation in the basolateral complex of the amygdala enhances discrimination between discrete cues and promotes configural processing of cues. Psychoneuroendocrinology 96, 84-92 (2018).

52. Jovanovic, T. et al. Fear potentiation is associated with hypothalamicpituitary-adrenal axis function in PTSD. Psychoneuroendocrinology 35 846-857 (2010).

53. Grillon, C. et al. Increased anxiety during anticipation of unpredictable aversive stimuli in posttraumatic stress disorder but not in generalized anxiety disorder. Biol. Psychiatry 66, 47-53 (2009).

54. Eckstein, M. et al. Oxytocin for learning calm and safety. Int. J. Psychophysiol. 136, 5-14 (2018).

55. Asok, A. et al. Optogenetic silencing of a corticotropin-releasing factor pathway from the central amygdala to the bed nucleus of the stria terminalis disrupts sustained fear. Mol. Psychiatry 23, 914-922 (2018). 